\title{
Autopercepção de saúde de mulheres com excesso de peso
}

\author{
Self-perception of health of women with excess weight \\ Autopercepción de la salud de las mujerescon exceso de peso
}

Recebido: 11/11/2021 | Revisado: 19/11/2021 | Aceito: 21/11/2021 | Publicado: 02/12/2021

Cátia Suely Palmeira

ORCID: https://orcid.org/0000-0001-6328-8118

Escola Bahiana de Medicina e Saúde Pública, Brasil

E-mail: catia_palmeira@yahoo.com.br

Natalia Vieira de Jesus

ORCID: https://orcid.org 0000-0002-0696-4666

Escola Bahiana de Medicina e Saúde Pública, Brasil

E-mail: nataliavieira06@ hotmail.com

Jessica Cruz da Silva Monteiro

ORCID: https://orcid.org0000-0002-3810-4708

Escola Bahiana de Medicina e Saúde Pública, Brasil

E-mail: js.jessicamonteiro@outlook.com

Fernanda Santos Oliveira

ORCID: https://orcid.org0000-0003-4094-2302

Escola Bahiana de Medicina e Saúde Pública, Brasil

E-mail: nandaoliveira1989@outlook.com

Tassia Teles Santana de Macedo

ORCID: https://orcid.org 0000-0003-2423-9844

Escola Bahiana de Medicina e Saúde Pública, Brasil

E-mail: tassiateles85@gmail.com

Fernanda Carneiro Mussi

ORCID: https://orcid.org 0000-0003-0692-5912 Universidade Federal da Bahia, Brasil E-mail: femussi@uol.com.br

\begin{abstract}
Resumo
Objetivo: descrever a autopercepção de saúde de mulheres com excesso de peso e verificar a sua associação com variáveis sociodemográficas, clínicas e hábitos de vida. Estudo transversal realizado em ambulatório de referência em obesidade, em Salvador-BA, sendo 101 mulheres entrevistadas mediante instrumentos específicos e submetidas a avaliação clínica. Os dados foram analisados descritivamente e pela estatística inferencial. A média de idade foi de 47,8 anos ( $\mathrm{dp}=9,0$ anos). Predominaram raça/cor negra $(93,1 \%), 2^{\circ}$ grau de escolaridade $(57,4 \%)$, situação conjugal sem companheiro $(51,5 \%)$, renda mensal familiar $\leq$ três salários-mínimos $(87,0 \%)$, obesidade grau III $(31,7 \%)$, presença de 1 a 2 comorbidades $(62,4 \%)$, principalmente hipertensão arterial $(61,4 \%)$. Quanto à autopercepção de saúde, $55,4 \%$ a classificaram como regular, $24,8 \%$ boa e $11,9 \%$ ruim. Houve associação estatisticamente significante entre autopercepção de saúde e prática de atividade física, consumo de bebida alcoólica e identificação das consequências do excesso de peso para a vida. A elevada prevalência de autopercepção de saúde negativa é preocupante considerando que está fortemente associada à morbidade e mortalidade.
\end{abstract}

Palavras-chave: Obesidade; Mulher; Autopercepção de saúde; População adulta.

\begin{abstract}
Objective: to describe the self-perception of health of overweight women and to verify its association with sociodemographic, clinical and lifestyle variables. Cross-sectional study carried out in a reference obesity clinic in Salvador-BA, with 101 women interviewed using specific instruments and undergoing clinical evaluation. Data were analyzed descriptively and using inferential statistics. The mean age was 47.8 years (sd $=9.0$ years). Black race/color predominated $(93.1 \%)$, high school education $(57.4 \%)$, marital status without a partner $(51.5 \%)$, family monthly income $\leq$ three minimum wages (87.0\%), obesity grade III (31.7\%), presence of 1 to 2 comorbidities (62.4\%), mainly arterial hypertension (61.4\%). As for self-rated health, 55.4\% classified it as regular, $24.8 \%$ good and $11.9 \%$ poor. There was a statistically significant association between self-perceived health and physical activity, alcohol consumption and identification of the consequences of being overweight for life. The high prevalence of negative selfrated health is worrisome considering that it is strongly associated with morbidity and mortality.
\end{abstract}

Keywords: Obesity; Woman; Self-perception of health, Adult population. 


\begin{abstract}
Resumen
Objetivo: describir la autopercepción de salud de mujeres con sobrepeso y verificar su asociación con variables sociodemográficas, clínicas y de estilo de vida. Estudio transversal realizado en clínica de obesidad de referencia en Salvador-BA, con 101 mujeres entrevistadas con instrumentos específicos y evaluadas mediante exámenes clínicos. Los datos se analizaron de forma descriptiva y mediante estadística inferencial. La edad media fue de 47,8 años (de = 9,0 años). Predominó la raza / color negro (93,1\%), educación secundaria $(57,4 \%)$, estado civil sin pareja $(51,5 \%)$, ingreso familiar mensual $\leq$ tres salarios mínimos $(87,0 \%)$, obesidad grado III $(31,7 \%)$, presencia de 1 a 2 comorbilidades $(62,4 \%)$, principalmente hipertensión arterial $(61,4 \%)$. En cuanto a la autopercepción de la salud, el $55,4 \%$ la clasifica como regular, el 24,8\% buena y el 11,9\% mala. Verificado asociación estadísticamente significativa entre la salud autopercibida y la actividad física, el consumo de alcohol y la identificación de las consecuencias del sobrepeso de por vida. La alta prevalencia de salud autoevaluada negativa es preocupante considerando que está fuertemente asociada con la morbilidad y la mortalidad.
\end{abstract}

Palabras clave: Obesidad; Mujer; Autopercepción de la salud; Población adulta.

\title{
1. Introdução
}

A prevalência de sobrepeso e obesidade está aumentando em todo o mundo, sendo que o número de adultos obesos aumentou mais de sete vezes nos últimos 40 anos (World Health Organization, 2021). No Brasil, o excesso de peso atinge 53,9\% de mulheres maiores de 18 anos, aumentando com a idade até os 64 anos e diminuindo com o aumento da escolaridade (Brasil, 2020). Está associado a um aumento do risco cardiovascular, decorrente do próprio acúmulo de peso corporal, e ainda pelas comorbidades associadas, como hipertensão arterial, diabetes e resistência à insulina (Inoue et al., 2018)

O acúmulo de peso representa uma condição resultante de múltiplas interações complexas entre fatores genéticos e comportamentais, principalmente relacionados à ingestão e gasto de energia (Piaggi et al., 2018). O balanço energético positivo subjacente à obesidade é geralmente atribuído a numerosos mecanismos e incluem principalmente o consumo excessivo de energia e aos baixos níveis de gasto energético relacionado à atividade física. Fatores ambientais e culturais também desempenham um papel crucial para o desenvolvimento do excesso de peso, principalmente em sociedades com a economia de mercado baseada no crescimento do consumo (Hebebrand et al., 2014).

A literatura evidencia que o acúmulo de peso corporal está associado a um aumento do risco cardiovascular, diabetes mellitus, doença renal crônica, alguns tipos de cânceres e uma série de distúrbios musculoesqueléticos, decorrente do próprio acúmulo de peso corporal, e ainda pelas comorbidades associadas (Inoue et al., 2018)

Atualmente, o excesso de peso, especialmente a obesidade, é considerado a principal causa de incapacidade funcional, redução da qualidade e expectativa de vida e aumento da mortalidade (Bell et al., 2017). As complicações da obesidade são profundas e potencializam a diminuição da atividade física e comportamentos sedentários contribuindo para uma espiral interminável de obesidade - consequências para a saúde - comportamentos limitantes da saúde - aumento ou manutenção da obesidade. Os efeitos fisiológicos de longo prazo da obesidade são numerosos e potencializam-se mutuamente (Williams et al., 2015).

Estar acima do peso ou ser obeso também tem implicações negativas nos aspectos psicossociais, como a discriminação baseada na aparência, baixa autoestima e desvantagem no mercado de trabalho que repercutem no estado geral de saúde, em sintomas depressivos e ansiosos, na diminuição da sensação de bem-estar, em autoconceito negativo em saúde e aumento da sensação de inadequação social levando a degradação das relações interpessoais (Lee et al., 2020).

A presença de condições crônicas de saúde resulta em chances estatisticamente menores de relatar "boa saúde", contribuindo significativamente para uma pior percepção da saúde (Bonner et al., 2017). Segundo os autores, isso ocorre porque a autopercepção da saúde não é afetada apenas por problemas clínicos, mas também por como a doença afeta a vida cotidiana. Atualmente, a autopercepção da saúde é um indicador amplamente utilizado para avaliar o estado de saúde, pois reflete a percepção e avaliação individual da própria saúde, representando um bom preditor da condição de saúde atual e futura. Refere-se não só a dimensão funcional e de bem-estar da saúde, bem como à extensão da adaptação, atitudes e cuidados 
em relação a uma doença existente (Szeles et al., 2015). A percepção de uma pessoa sobre a saúde não sobrevém apenas das sensações físicas de dor e desconforto, mas sobretudo das consequências sociais e psicológicas da presença da enfermidade (Meireles et al., 2017).

No tocante ao excesso de peso são escassos os estudos que fazem esta avaliação. Diante do exposto, os objetivos deste estudo foram descrever a autopercepção de saúde de mulheres com excesso de peso e verificar a sua associação com variáveis sociodemográficas, clínicas e hábitos de vida.

\section{Metodologia}

Trata-se de estudo transversal (Rouquayrol \& Almeida-Filho, 2009), realizado em um ambulatório de referência em estudo e acompanhamento de pessoas com excesso de peso, em Salvador-BA.

Foram incluídas neste estudo as mulheres que atenderam os critérios de inclusão como idade superior a 18 anos e inferior a 60 anos e índice de massa corporal $\geq 25 \mathrm{~kg} / \mathrm{m}^{2}$. Foram excluídas aquelas que por alguma razão física não tinham condições de realizar as medidas antropométricas, fizeram cirurgia bariátrica, estavam em uso de medicação para perda de peso e não compareceram ao serviço há mais de um ano. Assim, a amostra final foi composta por 101 mulheres. A inclusão apenas de mulheres foi devido ao número reduzido de homens frequentando regularmente o programa podendo constituir-se em viés para o estudo.

Com o objetivo de padronizar os procedimentos de coleta de dados, as entrevistadoras foram treinadas para a aplicação dos instrumentos e aferição das medidas antropométricas. Um teste-piloto foi realizado para verificar a necessidade de aprimorá-los, melhorando a aplicabilidade e entendimento dos mesmos. Após a realização do pré-teste, alguns ajustes com relação a terminologias e forma de aplicação dos formulários foram realizados.

Os dados foram coletados em duas etapas. Na primeira aplicou-se por meio de entrevista um formulário estruturado sobre variáveis sociodemográficas, clínicas e hábitos de vida e, na segunda, realizou-se a aferição das medidas antropométricas. Todas as mulheres elegíveis para o estudo foram orientadas sobre os objetivos e procedimentos da pesquisa e sobre o Termo de Consentimento Livre e Esclarecido, assim como tiveram suas dúvidas esclarecidas.

As variáveis independentes do estudo foram as sociodemográficas (idade, raça/cor autodeclarada, escolaridade, estado civil, renda familiar mensal, situação laboral, ter sofrido discriminação social em razão do excesso de peso, considerar as consequências do excesso de peso para a vida); os hábitos vida (prática de atividade física e consumo de bebida alcoólica); e clínicas (índice de massa corporal (IMC), doenças crônicas diagnosticadas, número de comorbidades, tempo de acompanhamento no serviço e dificuldade para controlar o peso).

A autopercepção de saúde, variável dependente do estudo, foi avaliada por meio da pergunta contida no instrumento de qualidade de vida Short-Form-36 (SF-36): "Como você avalia o seu estado de saúde?", contendo cinco respostas padronizadas como alternativas: "muito ruim, ruim, regular, bom e muito bom". Posteriormente, essas alternativas iniciais de respostas foram dicotomizadas em: ruim/regular (muito ruim, ruim e regular) e boa/muito boa (muito boa e boa).

A raça/cor autodeclarada foi classificada em branca, preta, parda, amarela e indígena. Neste estudo, esta variável foi agrupada em negras (cor preta e parda) e não negras (cor branca) $)^{(11)}$. A variável escolaridade foi classificada em até $1^{\circ}$ grau (analfabeta, assina o nome, $1^{\circ}$ grau incompleto, $1^{\circ}$ grau completo), $2^{\circ}$ grau (incompleto ou completo) e $3^{\circ}$ grau (incompleto ou completo). Quanto ao estado civil, adotou-se a classificação sem companheiro (solteira/viúva/divorciada) e com companheiro (casada/união estável). A renda familiar mensal foi autorreferida, sendo considerado o somatório do ganho mensal de cada componente da família que exercia atividade laboral. O valor obtido foi dividido em salários-mínimos, posteriormente, a renda foi categorizada em $\leq 3$ e $>3$ salários. Para a situação laboral adotou-se atividade com remuneração (empregado, autônomo e 
aposentado com outra atividade remunerada) e atividade sem remuneração (do lar, desempregada, estudante e aposentada sem atividade remunerada). O tempo de acompanhamento no serviço foi obtido no prontuário e classificado em $\leq 3$ anos e $>3$ anos.

Os dados antropométricos foram coletados em consultórios do ambulatório. Para a verificação do peso, as participantes foram orientadas a permanecerem em posição ortostática, de frente para o mostrador da balança, mantendo os braços ao longo do corpo e olhar fixo no horizonte, utilizando roupas leves e descalças. O peso (em quilogramas) foi apurado através de uma balança digital Scale, Model TEC 30 da marca Techline, com variação de $0,1 \mathrm{Kg}$. A altura (em metros) foi verificada por meio de um estadiômetro portátil (graduado a cada $0,5 \mathrm{~cm}$ ). Para esta medida a participante permaneceu de pé, com a cabeça e o dorso encostados na régua do estadiômetro, abaixo da haste horizontal, mantendo os braços esticados ao longo do corpo, ombros, omoplatas, nádegas, calcanhares encostados a parede e os pés apoiados no chão. Os valores do peso e da altura foram usados para determinar o índice de massa corpórea cuja fórmula é a razão entre o peso em quilogramas e o quadrado da altura em metros (IMC $=$ peso/altura ${ }^{2}$ ). Com o valor do IMC, as mulheres foram classificadas em: sobrepeso IMC $\geq 25 \mathrm{Kg} / \mathrm{m}^{2}$, Obesidade I IMC $=30,0$ a $34,9 \mathrm{Kg} / \mathrm{m}^{2}$, Obesidade II IMC $=35,0$ a $39,9 \mathrm{Kg} / \mathrm{m}^{2}$ e Obesidade III IMC $=\geq 40,0$ $\mathrm{Kg} / \mathrm{m}^{2}$ (World Health Organization, 2004)

Os dados constituíram um banco no Statistical Package for the Social Sciences (IBM SPSS versão 18.0). As variáveis categóricas foram analisadas em frequências absolutas e relativas e, as numéricas, em médias e desvio-padrão. Para verificar a associação entre a autopercepção de saúde e as variáveis de interesse empregou-se o teste Qui-quadrado de Pearson e o teste Exato de Fisher. Adotou-se significância estatística de 5\%.

O projeto foi aprovado pelo Comitê de Ética em Pesquisa da Escola de Enfermagem da Universidade Federal da Bahia (CAAE: 43665115.6.0000.5531) e parecer de ${ }^{\circ}$ 1.152.259, atendendo as exigências da resolução 466/2012 do Conselho Nacional de Saúde.

\section{Resultados}

Foram entrevistadas 101 mulheres, sendo que dessas, 6 (5,9\%) relataram uma autopercepção de saúde muito ruim, 12 $(11,9 \%)$ ruim, $56(55,4 \%)$ regular, $25(24,8 \%)$ boa e $2(2,0 \%)$ muito boa. Após transformação em uma variável dicotômica 74 $(73,3 \%)$ consideraram a sua saúde como muito ruim/ruim e 27 (26,8\%) como muito boa/boa.

A média de idade das participantes foi de 47,8 anos, desvio padrão de 9,0, com maior proporção na faixa etária entre 51 a 60 anos $(46,5 \%)$. Quanto às demais características sociodemográficas predominaram a raça/cor autodeclarada negra $(93,1 \%), 2^{\circ}$ grau completo e incompleto (57,4\%), situação conjugal sem companheiro (51,5\%), atividade laboral remunerada $(54,4 \%)$, renda familiar menor do que três salários-mínimos $(87,0 \%)$. Não foi encontrada associação significativa entre a autopercepção de saúde e variáveis sociodemograficas (Tabela 1). 
Tabela 1. Associação entre variáveis sociodemográficas e autopercepção de saúde de mulheres com excesso de peso, Salvador, 2016.

\begin{tabular}{|c|c|c|c|c|}
\hline \multirow[t]{2}{*}{ Variáveis } & \multirow[t]{2}{*}{ Total } & \multicolumn{2}{|c|}{ Autopercepção de saúde } & \multirow[b]{2}{*}{ Valor de $p$} \\
\hline & & $\begin{array}{c}\begin{array}{c}\text { Muito boa /Boa } \\
(n=27)\end{array} \\
\end{array}$ & $\begin{array}{c}\text { Muito ruim/ruim } \\
(n=74)\end{array}$ & \\
\hline Faixa etária & & & & 0,383 \\
\hline $20-30$ & $5(5,0 \%)$ & $0(0,0 \%)$ & $5(100,0 \%)$ & \\
\hline $31-40$ & $17(16,8 \%)$ & $6(35,3 \%)$ & $11(64,7 \%)$ & \\
\hline $41-50$ & $32(31,7 \%)$ & $10(31,3 \%)$ & $22(68,7 \%)$ & \\
\hline $51-60$ & $47(46,5 \%)$ & $11(23,4 \%)$ & $36(76,6 \%)$ & \\
\hline Cor/raça autodeclarada & & & & 1,000 \\
\hline Branca & $7(6,9 \%)$ & $2(28,6 \%)$ & $5(71,4 \%)$ & \\
\hline Negra (preta e parda) & $94(93,1 \%)$ & $27(26,7 \%)$ & $74(73,3 \%)$ & \\
\hline Escolaridade & & & & 0,595 \\
\hline Até $1^{\circ}$ grau & $30(29,7 \%)$ & $9(30,0 \%)$ & $21(70,0 \%)$ & \\
\hline $2^{\circ}$ grau completo e incompleto & $58(57,4 \%)$ & $16(27,6 \%)$ & $42(72,4 \%)$ & \\
\hline $3^{\circ}$ grau completo e incompleto & $13(12,9 \%)$ & $2(15,4 \%)$ & $11(84,6 \%)$ & \\
\hline Situação conjugal & & & & 0,964 \\
\hline Com companheiro & $49(48,5 \%)$ & $13(26,5 \%)$ & $36(73,5 \%)$ & \\
\hline Sem companheiro & $52(51,5 \%)$ & $14(26,9 \%)$ & $38(73,1 \%)$ & \\
\hline Situação laboral & & & & 0,558 \\
\hline Com atividade remunerada & $55(54,5 \%)$ & $16(29,1 \%)$ & $39(70,9 \%)$ & \\
\hline Sem atividade remunerada & $46(45,5 \%)$ & $11(23,9 \%)$ & $35(76,1 \%)$ & \\
\hline Renda familiar & & & & 0,095 \\
\hline$\leq 3$ salários-mínimos & $87(87,0 \%)$ & $21(24,1 \%)$ & $66(75,9 \%)$ & \\
\hline > 3 salários-mínimos & $13(13,0 \%)$ & $6(46,2 \%)$ & $7(53,8 \%)$ & \\
\hline
\end{tabular}

Fonte: Autores.

A Tabela 2 apresenta os resultados das análises bivariadas entre autopercepção de saúde e as variáveis independentes referentes aos hábitos de vida, as variáveis clínicas e as relacionadas ao tratamento e consequências do excesso de peso. A maioria das mulheres não realizava atividade física regular no lazer (62,4\%), e não consumia bebida alcoólica (64,4\%). Foi observado associação significante entre estas variáveis e autopercepção de saúde, notando-se pior percepção de saúde para as mulheres que não realizam atividade física e não consumiam bebida alcoólica. Em relação às variáveis clínicas, notou-se maior percentual de mulheres com IMC compatível com obesidade grau III (31,7\%), seguido de obesidade grau I (30,7\%).

As comorbidades mais frequentes foram: hipertensão arterial (61,4\%), diabetes mellitus tipo 2 (32,7,0\%), dislipidemia $(38,6 \%)$ e artrose $(32,7,0 \%)$. Sobre o número de comorbidades, 62,4\% agregaram diagnóstico médico de até duas dessas doenças crônicas. Não foi encontrada associação estatística entre classificação do excesso de peso, presença e número de comorbidades e autopercepção de saúde (Tabela 2).

Ainda na Tabela 2, observa-se que 84,2\% das participantes tinham dificuldade para seguir as recomendações para controlar o peso, metade $(50,0 \%)$ tinha tempo de acompanhamento no serviço maior que três anos, $83,2 \%$ relataram que o excesso de peso trouxe consequências para a vida e 64,4\% mencionaram não ter sofrido discriminação em função do peso. Apenas a variável considerar que o excesso de peso trouxe consequências para a vida mostrou associação significante com a autopercepção de saúde, notando-se que aquelas que acreditavam que o excesso de peso acarretava consequências negativas para a sua vida tiveram pior autopercepção de saúde. 
Tabela 2. Associação entre hábitos de vida, variáveis clínicas e relacionadas ao tratamento e a autopercepção de saúde das mulheres com excesso de peso, Salvador, 2016.

\begin{tabular}{|c|c|c|c|c|}
\hline \multirow[t]{2}{*}{ Variáveis } & \multirow[t]{2}{*}{ Total } & \multicolumn{2}{|c|}{ Autopercepção de saúde } & \multirow[t]{2}{*}{ Valor de p } \\
\hline & & Muito boa/Boa & Muito ruim/Ruim & \\
\hline \multicolumn{5}{|l|}{ Prática de atividade física } \\
\hline Sim & $38(37,6 \%)$ & $16(42,1 \%)$ & $22(57,9 \%)$ & $0,007 *$ \\
\hline Não & $63(62,4 \%)$ & $11(17,5 \%)$ & $52(82,5 \%)$ & \\
\hline \multicolumn{5}{|l|}{ Consumo de bebida alcoólica } \\
\hline Sim & $36(35,6 \%)$ & $1541,7 \%)$ & $21(58,3 \%)$ & $0,012 *$ \\
\hline Não & $65(64,4 \%)$ & $12(18,5 \%)$ & $53(81,5 \%)$ & \\
\hline \multicolumn{5}{|l|}{ IMC } \\
\hline Sobrepeso & $15(14,9 \%)$ & $6(40,0 \%)$ & $9(60,0 \%)$ & 0,636 \\
\hline Obesidade I & $31(30,7 \%)$ & $8(25,8 \%)$ & $23(74,2 \%)$ & \\
\hline Obesidade II & $23(22,8 \%)$ & $5(21,7 \%)$ & $18(78,3 \%)$ & \\
\hline Obesidade III & $32(31,7 \%)$ & $8(25,0 \%)$ & $24(75,0 \%)$ & \\
\hline \multicolumn{5}{|l|}{ Hipertensão arterial } \\
\hline Não & $39(38,6 \%)$ & $10(25,6 \%)$ & $29(74,4 \%)$ & \\
\hline Sim & $62(61,4 \%)$ & $17(27,4 \%)$ & $45(72,6 \%)$ & 0,844 \\
\hline \multicolumn{5}{|l|}{ Diabetes } \\
\hline Não & $68(67,3 \%)$ & $20(29,4 \%)$ & $48(70,6 \%)$ & 0,382 \\
\hline Sim & $33(32,7 \%)$ & $7(21,2 \%)$ & $26(78,8 \%)$ & \\
\hline \multicolumn{5}{|l|}{ Artrose } \\
\hline Não & $68(67,3 \%)$ & $19(27,9 \%)$ & $49(72,1 \%)$ & 0,694 \\
\hline Sim & $33(32,7 \%)$ & $8(24,2 \%)$ & $25(75,8 \%)$ & \\
\hline \multicolumn{5}{|l|}{ Número de comorbidades } \\
\hline Nenhuma & $15(14,9 \%)$ & $3(20,0 \%)$ & $12(80,0 \%)$ & 0,336 \\
\hline $1-2$ & $63(62,4 \%)$ & $20(31,7 \%)$ & $42(68,3 \%)$ & \\
\hline $3-4$ & $23(22,8 \%)$ & $4(17,4 \%)$ & $19(82,3 \%)$ & \\
\hline \multicolumn{5}{|l|}{ Dificuldade para controlar o peso } \\
\hline Sim & $85(84,2 \%)$ & $20(23,5 \%)$ & $65(76,5 \%)$ & 0,094 \\
\hline Não & $1615,8 \%)$ & $7(43,8 \%)$ & $9(56,3 \%)$ & \\
\hline \multicolumn{5}{|l|}{ Tempo de acompanhamento } \\
\hline$>3$ anos & $50(50,0 \%)$ & $16(32,0 \%)$ & $34(68,0 \%)$ & 0,171 \\
\hline$\leq 3$ anos & $50(50,0 \%)$ & $10(20,0 \%)$ & $40(80,0 \%)$ & \\
\hline $\begin{array}{l}\text { Consequências do excesso de peso para } \\
\text { a vida }\end{array}$ & & & & $0,038^{*}$ \\
\hline Sim & $84(83,2 \%)$ & $19(22,6 \%)$ & $65(77,4 \%)$ & \\
\hline Não & $17(16,8 \%)$ & $8(47,1 \%)$ & $9(52,9 \%)$ & \\
\hline Discriminação social & & & & 0,860 \\
\hline Sim & $36(35,6 \%)$ & $10(27,8 \%)$ & $26(72,2 \%)$ & \\
\hline Não & $65(64,4 \%)$ & $17(26,2 \%)$ & $48(73,8 \%)$ & \\
\hline
\end{tabular}

Fonte: Autores.

\section{Discussão}

Este estudo mostrou que a maioria das participantes não tinha uma boa autopercepção de saúde, o que pode ser explicado pelo fato da obesidade por ser uma doença crônica relacionada ao desenvolvimento de comorbidades com importantes repercussões físicas e psicossociais, prejudicando não apenas o estado de saúde objetivo da pessoa, mas também a sua autopercepção de saúde (Busutil et al., 2017). Vale destacar que pessoas que se consideram menos saudáveis apresentam uma definição menos holística de saúde e mostram-se menos capazes de usar seus recursos para realizar as atividades da vida diária, melhorar os hábitos alimentares e a prática de exercícios físicos no lazer e ainda lidar com os estressores da vida (Rathbun, Loerzel \& Edwards, 2020).

Neste estudo, a média de idade de 47,8 anos e a predominância de mulheres com menor renda representam resultados esperados, pois a frequência do excesso de peso na população brasileira é maior com o aumento da idade (Brasil. Ministério da Saúde. (2020). O maior percentual de mulheres na faixa etária de 50 a 60 anos que perceberam sua saúde como "ruim" pode 
relacionar-se ao incremento da idade que aumenta a probabilidade das pessoas terem um maior número de comorbidades, o que pode contribuir na autopercepção negativa de saúde (Bell, Sabia, Singh-Manoux, Hamer \& Kivimäki, 2017). Resultados de estudos confirmaram que a idade avançada estava associada à pior autopercepção de saúde (51-65 anos vs. 35-50 anos OR = 2,1, IC 95\% = 1,7- 2,6) (Kraja, Kraja, Cakerri \& Burazeri, 2016) e mulheres adultas e idosas percebem de maneira mais negativa a sua saúde do que os homens, e que quanto mais velho o grupo etário, maior a prevalência de autopercepção regular/ruim de saúde (Reichert, Loch \& Capilheira, 2012).

Considerando que a idade da maioria das entrevistadas correspondia ao período de pós-menopausa e que nesta fase mudanças físicas, hormonais e psicossociais estão presentes de forma simultânea, um declínio no estado de saúde bem como a redução na condição das pessoas em realizar atividades cotidianas, causam efeito na percepção de saúde dos indivíduos (Silva, Rocha \& Caldeira, 2018).

O maior percentual de mulheres negras está de acordo com o perfil da população de Brasileira, pois segundo o Instituto Brasileiro de Geografia e Estatística (IBGE), a região Nordeste tem a maior proporção de pessoas declaradas da cor preta, e a segunda maior de cor parda (Instituto Brasileiro de Geografia e Estatística [IBGE], 2016). Apesar da ausência de associação entre cor/raça e percepção de saúde neste estudo, pessoas bancas apresentam em geral melhores indicadores de saúde devido a sua relação com uma melhor condição social e econômica, e assim mais acesso a bens e serviços de saúde (Reichert, Loch, \& Capilheira, 2012).

$\mathrm{O}$ pequeno número de mulheres com maior grau de escolaridade reflete a população acompanhada no centro de referência onde foi realizada a pesquisa, o qual atende prioritariamente a população oriunda do SUS e que possui baixa condição socioeconômica. Não obstante não tenha sido verificada associação significativa entre esta variável e autopercepção de saúde no presente estudo, existem evidências de forte associação entre elas na literatura (Kraja, Kraja, Cakerri, \& Burazeri, 2016). A escolaridade é um indicador estreitamente relacionado a emprego, renda, posse de bens e acesso a serviços, aspectos socioeconômicos capazes de prejudicar a saúde percebida (Cialani \& Mortazavi, 2020). De acordo com os autores, o ensino superior ajuda as pessoas a melhorarem suas habilidades para o alcance dos objetivos de vida e ter um estilo de vida saudável.

No que tange a situação laboral remunerada, mesmo sendo em menor frequência, merece destaque que 45,5\% não tinham atividade remunerada. Achados de estudo mostraram que pessoas sem ocupação, quando comparadas a aquelas empregadas tiveram 5,6 vezes mais chances de avaliar seu estado de saúde como ruim, e que o desemprego de longa duração pode prejudicar a saúde mental ou causar problemas de saúde (Kraja, Kraja, Cakerri, \& Burazeri, 2016). Não poder trabalhar ou a própria condição de desemprego podem causar algum tipo de sofrimento, levando a ausência de um trabalho formal a contribuir para uma percepção negativa do estado de saúde (Silva, Rocha, \& Caldeira, 2018).

A predominância de mulheres com renda familiar menor ou igual a três salários-mínimos confirma o perfil da população constituída da maioria de pessoas com baixo poder aquisitivo. Mesmo não tendo associação estatística entre essa variável e autopercepção de saúde, considera-se que o impacto psicossocial de pertencer a uma classe social específica influencia a saúde física dos indivíduos e a forma como percebem a sua saúde, pois reflete a capacidade dos indivíduos de atender às suas necessidades e a falta de recursos econômicos relaciona-se a maior probabilidade de ter uma percepção de saúde insatisfatória (Cialani \& Mortazavi, 2020). Ser membro de uma família com privação material aumenta as chances de perceber a saúde de forma negativa (López Del Amo González, Benítez \& Martín-Martín, 2018).

Mesmo sem diferença estatisticamente significante e não representar a maioria das participantes, sofrer discriminação pode envolver sentimentos negativos tais como, medo, raiva, tristeza, vergonha, frustração, baixa autoestima e desânimo, o que pode levá-las ao sofrimento, instabilidade emocional, a ansiedade e até depressão (Palmeira, Santos, Mussi \& Silva, 2020), condições estas que pode interferir na forma como as pessoas percebem sua saúde e realizam práticas de autocuidado. 
Com relação aos hábitos de vida, verificou-se no presente estudo que a prática de atividade física no lazer e o consumo de bebida alcoólica estiveram associados à autopercepção do estado de saúde. Igualmente em estudo realizado na Colombia, verificou-se associação entre atividade física e estado de saúde autoavaliado, apontando que mulheres fisicamente inativas apresentaram maior chance para perceber sua saúde como ruim e regular (OR 1,30, IC95\% 1,03, 1,62) (MendozaRomero, Urbina, Cristancho-Montenegro \& Rombaldi, 2019). Pesquisa desenvolvida no Brasil afirma que menor prática de atividade física se associa a piores níveis de autopercepção de saúde, e ainda que a prática de atividade física contribui de forma benéfica para a saúde aumentando a sensação de bem-estar físico e mental, o que pode levar os indivíduos a uma melhor autopercepção de saúde (Andrade, Loch \& Silva, 2019).

Uma maior frequência de mulheres que não realizavam atividade física no lazer verificada no presente estudo é preocupante, considerando esta prática é preconizada como tratamento para o controle do peso, e também por representar uma escolha extremamente benéfica para a saúde, pois aumenta a expectativa de vida, promove bem-estar, reduz o estresse, a ansiedade, a depressão, a prevalência de doenças cardiovasculares, diabetes, câncer, em última instância melhora a qualidade de vida e, consequentemente, influencia na autopercepção de saúde do indivíduo (Silva, Rocha \& Caldeira, 2018; World Health Organization, 2020). Evidencias na literatura apontam que a prática regular de atividade física contribui a manutenção da independência e autonomia das pessoas e, consequentemente, para o bem-estar biopsicossocial (Silva, Rocha \& Caldeira, 2018).

Com relação à associação entre consumo de bebida alcoólica e autopercepção de saúde, uma possível razão pela qual esse consumo foi relacionado à melhor autopercepção da saúde é que pessoas que se sentem saudáveis são mais frequentemente consumidoras de álcool, em comparação aquelas que se sentem doentes (Kraja, Kraja, Cakerri \& Burazeri 2016).

Concernente ao IMC, os resultados revelaram que mulheres com obesidade grau II e III predominam no grupo pesquisado. Este dado merece destaque, pois é sabido que quanto maior o grau de obesidade maior o risco de complicações por outras doenças crônicas. Estas condições sobrecarregam especialmente as mulheres não saudáveis, fazendo-as se sentirem definidas por suas doenças, embora diferindo entre a composição corporal e os níveis socioeconômicos, especialmente o nível de educação e a renda familiar (Rathbun, Loerzel \& Edwards, 2020).

Estudo aponta que pessoas com sobrepeso e obesidade relataram saúde ruim com mais frequência do que aquelas com peso normal (Tang, Zhao \& Li, 2017). Ainda de acordo com os autores esta percepção ruim da saúde é maior em mulheres também devido ao fato de que quase todas elas enfrentam maiores pressões sociais, como discriminação de peso e problemas de imagem corporal, independentemente do nível socioeconômico. Estar com sobrepeso tem um impacto insignificante na autopercepção de saúde, enquanto ser obeso tem um impacto negativo estatisticamente significante, sendo que esses efeitos são mais pronunciados para aqueles que têm um maior grau de obesidade (Cullinan \& Gillespie, 2016). Mesmo sem significância estatística, observou-se nesse estudo, maior percentual de percepção ruim de saúde para mulheres com obesidade em relação aquelas com sobrepeso.

Ainda que não tenha sido encontrada significância estatística entre presença e número de comoborbidades neste estudo, sabe-se que a presença de doenças crônicas pode implicar em uma maior probabilidade de se avaliar a saúde de forma negativa. Essa associação negativa é esperada, sobretudo, entre as pessoas mais velhas, podendo refletir a concepção de que ter saúde é não ter doenças (Silva, Rocha \& Caldeira, 2018). De acordo com o autor a presença de comorbidades pode implicar em limitações para atividades cotidianas e também para o autocuidado. Estudo sobre autopercepção da saúde entre adultos e idosos usuários da atenção básica de saúde verificou maior prevalência de autopercepção negativa naqueles com maior número de doenças crônicas (Lindemann, Reis, Mintem \& Mendoza, 2019). Tendo consciência desses achados é importante conhecer 
essas condições e, assim, poder prevenir os possíveis agravos através de estratégias de assistência e educação para o autocuidado para esta população.

Resultado de estudo qualitativo apontou que mulheres que não se sentiam saudáveis tinham o dobro de comorbidades em comparação com as mulheres que se percebiam como saudáveis, e que as doenças mais comumente referidas eram a hipertensão arterial, a hiperlipidemia e o excesso de peso, e que uma mulher que referiu sentir-se não saudável contou como se sentiu consumida por suas doenças (Rathbun, Loerzel \& Edwards, 2020). Por outro lado, é preocupante também o fato de parte das entrevistadas, com presença de 3 a 4 comorbidades, terem referido sua saúde como boa, pois esta percepção também pode contribuir para a não compreensão da necessidade de modificar fatores de estilo de vida que melhorariam saúde, como a implementação de uma dieta mais saudável e exercícios (Dalbo,Teramoto, Roberts \& Scanlan, 2017).

Independentemente de não se ter sido verificado associação entre autopercpção de saúde e hipertensão arterial e diabetes mellitus autorreferida, no presente estudo, destaca-se que estas doenças podem interferir na forma como as pessoas percebem a sua saúde. Dados resultantes de pesquisa evidenciaram que ter o diagnóstico de diabetes ou hipertensão arterial aumentou a percepção de saúde insatisfatória em $62,0 \%$, enquanto para os que tinham dois agravos, a percepção negativa da saúde foi duas vezes maior quando comparados àqueles que não tinham nenhuma dessas doenças (Lima et al., 2021).

A relação entre diabetes autorreferida e autopercepção negativa de saúde também foi evidenciada em pesquisa realizada em cidade do interior do nordeste do Brasil, no qual o autor sugeriu que esta associação pode ser ocasionada pelas limitações ou incapacidades na realização das atividades cotidianas, da dificuldade de controle da doença e das restrições alimentares (Santos, Coqueiro \& Henrique, 2016). Em nosso estudo, não se constatou significância estatística entre diabetes e autopercepção de saúde, embora tenha-se observadao maior percentual as mulheres diabéticas com pior autopercepção de saúde.

Considerando que entre as complicações da obesidade estão os distúrbios músculo esqueléticos, incluindo a artrose, e que esta que se caracteriza por dor, rigidez articular, edemas, deformidades e incapacidade de movimentos (Bussière, Sicsic \& Pelletier-Fleury, 2014) e mesmo que somente cerca de um terço tenha referido ter este problema de saúde, sem associação significante com a autopercepção de saúde, esse achado merece ser ressaltado. As manifestações clínicas da artrose além provocar sofrimento e impedir que a pessoa pratique atividade física necessária ao controle do peso, pode levá-la a perceber sua saúde de forma negativa.

Destaca-se que o grande percentual de mulheres que referiram dificuldade para controlar o peso e que tinham autopercepção de saúde ruim é preocupante, pois esta dificuldade pode fazer com que as mesmas se sintam desmotivadas para continuar o tratamento, o que pode agravar ainda mais os danos à saúde. Quanto ao tempo de acompanhamento no serviço, maior percentual das que percebiam sua saúde de forma negativa tinham até três anos. Pode-se inferir que o fato de já se ter um acompanhamento mais longo pela equipe do serviço pode ter ajudado algumas mulheres a enfrentar os problemas de saúde de forma mais positiva.

Este estudo apresenta algumas limitações que precisam ser ponderadas na interpretação e generalização dos resultados. O delineamento transversal não permite que sejam feitas inferências de causa-efeito em relação às variáveis estudadas e a pesquisa foi realizada em um único cenário. Outra possível limitação se refere a viés de relato e dificuldades de interpretação da medida de avaliação da autopercepcão de saúde que pode, também, apresentar outros resultados em diferentes grupos culturais e socioeconômicos. Por outro lado, destaca-se a relevância do presente estudo cujos resultados apresentados representam informações importantes que podem contribuir para reflexões mais aprofundadas sobre a autopercepção de saúde e fatores associados e criação de estratégias de intervenções para melhorar a autoperceção de saúde de mulheres com excesso de peso. Sem dúvida o estudo contribui para o avanço do conhecimento sobre o objeto sob investigação. 


\section{Conclusão}

Os resultados deste estudo destacam que a autopercepção de saúde das mulheres com excesso de peso foi considerada ruim (regular, ruim e muito ruim) pela maioria delas. Houve associação estatisticamente significante entre autopercepção de saúde com prática de atividade física, consumo de bebida alcoólica e identificação das consequências do excesso de peso para a vida. A elevada prevalência de autopercepção de saúde negativa é preocupante considerando esta variável está fortemente associada à morbidade e mortalidade.

Nesse sentido, é de suma importância o reconhecimento de tais aspectos especialmente pelos profissionais de saúde que prestam cuidados as pessoas com excesso de peso, no sentido da utilização de abordagens ampliadas que ajudem as usuárias dos serviços a enfrentar a doença e tratamento dos seus problemas de saúde, a desenvolverem práticas de autocuidados voltadas para os aspectos biopsicossociais do processo de adoecimento. Pensa-se ainda que há muito a ser discutido sobre o tema, portanto se faz necessário que novas pesquisas sejam realizadas com amostras maiores e abordando mais variáveis.

\section{Agradecimentos}

O trabalho contou com financiamento do Programa Institucional de Bolsas de Iniciação Científica da Fundação de Amparo à Pesquisa e Inovação do Estado da Bahia (FAPESB) - 2015/2016 e de auxílio financeiro do CNPQ, processo no 421599/2016-2.

\section{Referências}

Andrade, G. F., Loch, M. R., \& Silva, A. M. R. (2019). Mudanças de comportamentos relacionados à saúde como preditores de mudanças na autopercepção de saúde: estudo longitudinal (2011-2015). Cadernos de Saúde Pública. 35(4), e00151418. https://doi.org/10.1590/0102-311X00151418.

Bell, J., Sabia, S., Singh-Manoux, A., Hamer, M., \& Kivimäki, M. (2017). Healthy obesity and risk of accelerated functional decline and disability. Int J Obes. 41, 866-872. https://doi.org/10.1038/ijo.2017.51

Bonner, W., Weiler, R., Orisatoki, R., Lu, X., Andkhoie, M., Ramsay, D., Yaghoubi, M., Steeves, M., Szafron, M., \& Farag, M. (2017). Determinants of selfperceived health for Canadians aged 40 and older and policy implications. International journal for equity in health, 16(1), 94. https://doi.org/10.1186/s12939017-0595-x

Brasil. Ministério da Saúde. (2020). Vigitel Brasil 2019: vigilância de fatores de risco e proteção para doenças crônicas por inquérito telefônico: estimativas sobre frequência e distribuição Secretaria de Vigilância em Saúde. Departamento de Análise em Saúde e Vigilância de Doenças Não Transmissíveis. Vigitel

Brasil 2019: vigilância de fatores de risco e proteção para doenças crônicas por inquérito telefônico: estimativas sobre frequência e distribuição sociodemográfica de fatores de risco e proteção para doenças crônicas nas capitais dos 26 estados brasileiros e no Distrito Federal em 2019 - Brasília: Ministério da Saúde, 137

Bussière, C., Sicsic, J. \& Pelletier-Fleury, N. (2014). The effects of obesity and mobility disability in access to breast and cervical cancer screening in France: results from the National Health and Disability Survey. BMC Health Serv Res 14, P13. https://doi.org/10.1186/1472-6963-14-S2-P13

Busutil, R., Espallardo, O., Torres, A., Martínez-Galdeano, L., Zozaya, N., \& Hidalgo-Vega, Á. (2017). The impact of obesity on health-related quality of life in Spain. Health Qual Life Outcomes. 10;15(1),197. 10.1186/s12955-017-0773-y.

Chaput, J. P., Pérusse, L., Despré,s J. P., Tremblay, A., \& Bouchard, C. (2014). Findings from the Quebec Family Study on the Etiology of Obesity: Genetics and Environmental Highlights. Current obesity reports. 3(1), 54-66. https://doi.org/10.1007/s13679-013-0086-3

Cialani, C., Mortazavi, R. (2020). The effect of objective income and perceived economic resources on self-rated health. Int J Equity Health. 19, 196 https://doi.org/10.1186/s12939-020-01304-2

Cullinan, J., \& Gillespie, P. (2016). Does Overweight and Obesity Impact on Self-Rated Health? Evidence Using Instrumental Variables Ordered Probit Models. Health economics. 25(10):1341-8. 10.1002/hec.3215

Dalbo, V. J., Teramoto, M., Roberts, M. D., \& Scanlan, A. T. (2017). Lack of Reality: Positive Self-Perceptions of Health in the Presence of Disease. Sports (Basel, Switzerland), 5(2), 23. https://doi.org/10.3390/sports5020023

Hebebrand, J., Holm, J. C., Woodward, E., Baker, J. L., Blaak, E., Durrer, S. D., Farpour-Lambert, N. J., Frühbeck, G., Halford, J. G. C., Lissner, L., Micic, D., Mullerova, D., Roman, G., Schindler, K., Toplak, H., Visscher, T. L. S., \& Yumuk, V. (2017). A Proposal of the European Association for the Study of Obesity to Improve the ICD-11 Diagnostic Criteria for Obesity Based on the Three Dimensions Etiology, Degree of Adiposity and Health Risk. Obes Facts. 10(4),284-307. 10.1159/000479208. 
Inoue, Y., Qin, B., Poti, J., Sokol, R., \& Gordon-Larsen, P. (2018). Epidemiology of Obesity in Adults: Latest Trends. Curr Obes Rep. 7(4),276-8. $10.1007 / \mathrm{s} 13679-018-0317-8$.

Instituto Brasileiro de Geografia e Estatística - IBGE (2016). Pesquisa nacional por amostra de domicílios: síntese de indicadores 2015 / IBGE, Coordenação de Trabalho e Rendimento. - Rio de Janeiro, 2016. 108p. https://biblioteca.ibge.gov.br/visualizacao/livros/liv98887.pdf

Kraja, F., Kraja, B., Cakerri, L., \& Burazeri, G (2016). Socio-demographic and lifestyle correlates of self-perceived health status in a population-based sample of Albanian adult men and women. Mater Sociomed. 28(3),173-7. 10.5455/msm.2016.28.173-177.

Lee, H., Ahn, R., Kim, T. H., \& Han, E. (2019). Impact of Obesity on Employment and Wages among Young Adults: Observational Study with Panel Data. Int. J. Environ. Res. Public Health. 16, 139. 10.3390/ijerph16010139

Lindemann, I. L., Reis, N. R., Mintem, G. C., \& Mendoza, R.A.M. (2019). Autopercepção da saúde entre adultos e idosos usuários da Atenção Básica de Saúde. Cien Saude Colet. 24 (1), 45-52. https://doi.org/10.1590/1413-81232018241.34932016.

López Del Amo González, M. P., Benítez, V., \& Martín-Martín, J. J. (2018). Long term unemployment, income, poverty, and social public expenditure, and their relationship with self-perceived health in Spain (2007-2011). BMC Public Health. 18(1):133. 10.1186/s12889-017-5004-2

Meireles, A. L., Xavie,r C. C., Andrade, A. C. S., Friche, A. A. L., Proietti, F. A., \& Caiaffa, W. T. (2015). Self-rated health in urban adults, perceptions of the physical and social environment, and reported comorbidities: The BH Health Study. Cad Saúde Pública. 31 (Suppl:S1), 20-35.

Mendoza-Romero, D., Urbina, A., Cristancho-Montenegro, A., \& Rombaldi, A. (2019). Impact of smoking and physical inactivity on self-rated health in women in Colombia. Prev Med Rep. 16:100976. 10.1016/j.pmedr.2019.100976

Palmeira, C. S., Santos, L. S., Mussi, F. C., \& Silva, S. M. B. (2020). Stigma Perceived By Overweight Women. Rev Bras Enferm. 73(Suppl 4):e20190321. http://dx.doi.org/10.1590/0034-7167-2019-0321

Piaggi, P., Vinales, K.L., Basolo, A., Santini, F., \& Krakoff, J. (2018). Energy expenditure in the etiology of human obesity: spendthrift and thrifty metabolic phenotypes and energy-sensing mechanisms. J Endocrinol Invest. 41(1):83-89. 10.1007/s40618-017-0732-9

Puhl, R.M., Himmelstein, M.S., \& Pearl, R. L. (2020). Weight stigma as a psychosocial contributor to obesity. American Psychologist. 2020; 75(2), 274-289. https://doi.org/10.1037/amp0000538

Rathbun, K. P., Loerzel, V., \& Edwards, J. (2020). Personal Perception of Health in Urban Women of Low Socioeconomic Status: A Qualitative Study. Journal of primary care \& community health. 11, 2150132720925951. https://doi.org/10.1177/2150132720925951

Reichert, F. F., Loch, M.R., \& Capilheira, M. F. (2012). Autopercepção de saúde em adolescentes, adultos e idosos. Ciênc. saúde coletiva. 17(12), 3353-62. https://doi.org/10.1590/S1413-81232012001200020.

Rouquayrol, Z. M. \& Almeida-Filho, N. (2009). Epidemiologia e Saúde. Guanabara Koogan.

Santos, M. S., Coqueiro, R., \& Henrique, F.M. (2016). Estudo populacional sobre os determinantes da autopercepção de saúde de idosos residentes em comunidade. Ciencia y enfermería, 22(2), 13-26. https://dx.doi.org/10.4067/S0717-95532016000200002

Silva, V. H., Rocha, J. S. B., \& Caldeira, A. P. (2018). Fatores associados à autopercepção negativa de saúde em mulheres climatéricas. Ciênc. saúde coletiva. 2018; 23(5): 1611-1620. https://doi.org/10.1590/1413-81232018235.17112016

Szeles, M. R. (2018). Comparative Examination of Self-Perceived Health and Other Measures of the Quality of Life Across the EU-27, Social Indicators Research: An International and Interdisciplinary Journal for Quality-of-Life Measurement, Springer. 137(1):391-411. 10.1007/s11205-017-1597-1

Tang, K., Zhao, Y. \& Li, C. (2017). The association between self-rated health and different anthropometric and body composition measures in the Chinese population. BMC Public Health. 17, 317. https://doi.org/10.1186/s12889-017-4249-0

Williams, E. P., Mesidor, M., Winters, K., Dubbert, P. M., \& Wyatt, S. B. (2015). Overweight and Obesity: Prevalence, Consequences, and Causes of a Growing Public Health Problem. Curr Obes Rep. 4(3):363-70. 10.1007/s13679-015-0169-4. PMID: 26627494.

World Health Organization (WHO). (2004). Obesity: preventing and managing the global epidemic of obesity. Geneva. https://apps.who.int/iris/handle/10665/42330

World Health Organization (WHO). (2021). Obesity And Overweight. Fact Sheet. Disponivel: https://www.who.int/news-room/fact-sheets/detail/obesity-andoverweight

World Health Organization. (WHO). (2020). Guidelines on physical activity and sedentary behavior. https://www.who.int/publications/i/item/9789240015128 